\title{
Performance Analysis of Region based Hybrid Compression for Medical Images
}

\author{
Preeti V. Joshi \\ PG Student \\ Department of Electronics \\ and Telecommunication \\ V.E.S. Institute of Technology \\ Mumbai, India
}

\author{
C. D. Rawat \\ Associate Professor \\ Department of Electronics and \\ Telecommunication \\ V.E.S. Institute of Technology \\ Mumbai, India
}

\begin{abstract}
The high quality of the image produced by CT scan and MRI techniques is required to be maintained in order to avoid wrong diagnosis along with reduced file size. A lossless compression technique retains the image quality but achieves low to moderate compression ratio. Lossy compression, on the other hand, provides higher compression at the cost of degraded image quality. Thus there is need of intermediate method that can satisfy both the requirements. One such approach is the region based hybrid compression in which both lossless and lossy techniques are integrated to obtained better results. Present work comprises region based hybrid compression using Huffman coding and SPIHT. First the diagnostically important region is separated from the rest of the image by a segmentation procedure. The extracted ROI is coded using lossless Huffman coding and SPIHT compression is used for rest of the image also called as background. Performance of the proposed method is evaluated in terms of full reference and no reference parameters.
\end{abstract}

\section{Keywords}

BRISQUE, FSIM, Hybrid Compression, ROI, SPIHT, SSIM, VIF.

\section{INTRODUCTION}

In today's modern era, multimedia technology has tremendous impact on human lives. Image is one of the most important and largely used media contributing to multimedia. Images form a significant part of data, particularly in remote sensing, biomedical and video conferencing applications. This application involves transmission of images over the internet to acquire the information. Such processed data requires much more storage, high speed compute processor and much more bandwidth for transmission. Thus there is need to process an image by such technique that provides an efficient way of storage and transmission [1].

One of the methods that address this problem is image compression. Compression lessens the size of image. The goal of image compression is to represent an image with minimum bit rate by exploiting the redundancies present in it [2]. Redundancy refers to the amount of wasted space used in transmission [3]. The three basic redundancies in digital image processing are coding redundancy, interpixel redundancy and psychovisual redundancy [4]. Coding redundancy occurs when less than optimal code words are used. Interpixel redundancy corresponds to statistical dependencies among pixels, especially between neighboring pixels. Psychovisual redundancy occurs due to different sensitivities of human eye for different images. Compression is achieved by removal of one or more redundancies.

\subsection{Compression Techniques}

The image compression techniques are broadly classified into two categories: lossless and lossy depending whether or not an exact replica of the original image could be reconstructed using the compressed image. In lossless compression, the image after compression and decompression is identical to the original image and every bit of information is preserved during the decomposition process. The reconstructed image after compression is an exact replica of the original one [5]. Although lossless compression methods have the appeal that there is no deterioration in image quality, this scheme only achieves a modest compression rate. Some of the commonly used lossless compression methods are run Length Encoding, Huffman Coding, Arithmetic Encoding and Dictionary Based Encoding [6].

In lossy compression, the reconstructed image contains degradations with respect to the original image. The perfect reconstruction of the image is sacrificed by the elimination of some amount of redundancies in the image to achieve higher compression ratio [7]. The compression ratio is higher as compared to lossless compression. The term 'visually lossless' is often used to characterise lossy compression schemes that result in no visible degradation under a set of designated viewing conditions

A typical lossy image compression consists of three closely connected components namely source encoder, quantizer, and entropy encoder. Source coding is done by various transform techniques such as DFT, DCT which converts pixels in the original image into frequency domain coefficients. In fractal coding, the original image is forst segmented by parameters such as colour separation, edge detection and spectrum and texture analysis. Quantization involves many to one mapping. This can be performed by scalar or vector quantizers [8].

The techniques mentioned so far performs image compression in spatial domain. A discrete wavelet transform can also be used for compression in wavelet domain [9]. The wavelet transform decomposes an image into a set of different resolution sub-images, corresponding to the various frequency bands. This result in multiresolution representation of images with localization in both spatial and frequency domains [10]. This exploits the fact that the response of the human eye is different to high and low frequency components of an image. The commonly used wavelet based techniques are EZW, SPIHT, WDR, ASWDR algorithm [11].

\subsection{Medical Imaging}

Medical imaging is the technique, process and art of creating visual representations of the interior of a body for clinical analysis and medical intervention. Medical imaging attempts 
to reveal internal structures hidden by the skin and bones, as well as to diagnose and treat disease. Medical imaging also establishes a database of normal anatomy and physiology to make it possible to identify abnormalities [12] Imaging techniques extensively used in medicine include X-Ray, Magnetic Resonance Imaging (MRI), Ultrasound and Computed Tomography (CT). MRI does not use ionizing radiation and is therefore considered to be safer than many other imaging techniques. MRI generates images with excellent tissue contrast and thus is particularly used for neurological, cardiovascular and oncological imaging [13].

For the detection brain tumor, conventional MRI exploits physical properties of tissue protons such as density and relaxation time for longitudinal and transverse magnetization to generate signals that are imaged as areas of different contrast. Based on the type of contrast generated, the images are classified as $\mathrm{T} 1$ weighted, $\mathrm{T} 2$ weighted and proton density weighted MRI images. In all three types of images, bright areas correspond to tissue with high signal intensity and are referred to as hyperintense, whereas dark areas are referred to as hypointense that correspond to low signal intensity. The tumor portion appears hyperintense for $\mathrm{T} 1$ weighted images while it is hypointense for $\mathrm{T} 2$ weighted images as shown in figure 1 . In case of proton density weighted images, the hyperintense tumor is surrounded by hyperintense cells [14]. In the present work, $\mathrm{T} 1$ and $\mathrm{T} 2$ weighted MRI images are taken as test images.

The rest of the paper is organized as follows: section 2 explains the proposed methodology alongwith compression algorithm used. Section 3 covers the simulation results for various MRI images. The work is concluded in section 4

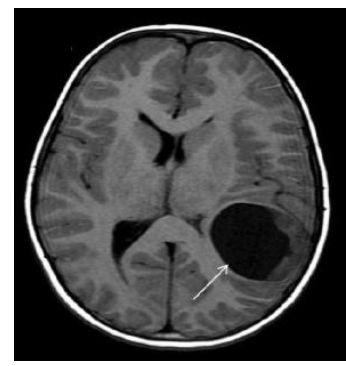

(a) T1 Weighted scan

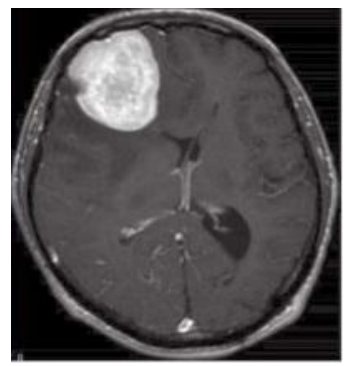

(b) T2 Weighted scan
Fig 1: Different types of MRI images

\section{PROPOSED METHOD}

Region based coding is form of selective image compression [15]. The MRI image is divided into regions based on their diagnostic importance. Region of Interest (ROI) is the diagnostically significant information i.e. the affected part of image used for analysis. It represents the tumor region in case of brain MRI images. The tumor is located over very small regions, nearly about $5-10 \%$ of the total area of the image. The rest of the image is termed as NON-ROI [16]. Hybrid compression combines lossy and lossless techniques to achieve higher compression at visually acceptable quality. Lossless compression is used for abnormal regions that are important for diagnosis and therefore achieving high quality, while NON-ROI region undergoes lossy compression [17]. The compression and decompression procedure for the proposed work is shown in Fig 2 and 3 respectively.

\subsection{ROI Extraction}

The tumor portion is separated from the rest of the image using a manual segmentation procedure. The window required for the selection of the tumor portion can be of any standard shapes such as rectangular, circular or polygonal. Rectangular window requires four corners to be defined around the desired region. The number of boundary points required by the user for selection of region is user defined in case of polygonal window. However, the complexity increases with increase in number of boundary points. A circular cropping window is used to select the tumor region. The window requires two inputs namely centre and the required radius. The locus use for cropping image in circular form is given by [18]

$$
(x-a)^{2}+(y-b)^{2}=R^{2}
$$

Where $(\mathrm{a}, \mathrm{b})$ are the $\mathrm{x}$ and $\mathrm{y}$ coordinates of the centre and $\mathrm{R}$ is the radius of the circle.

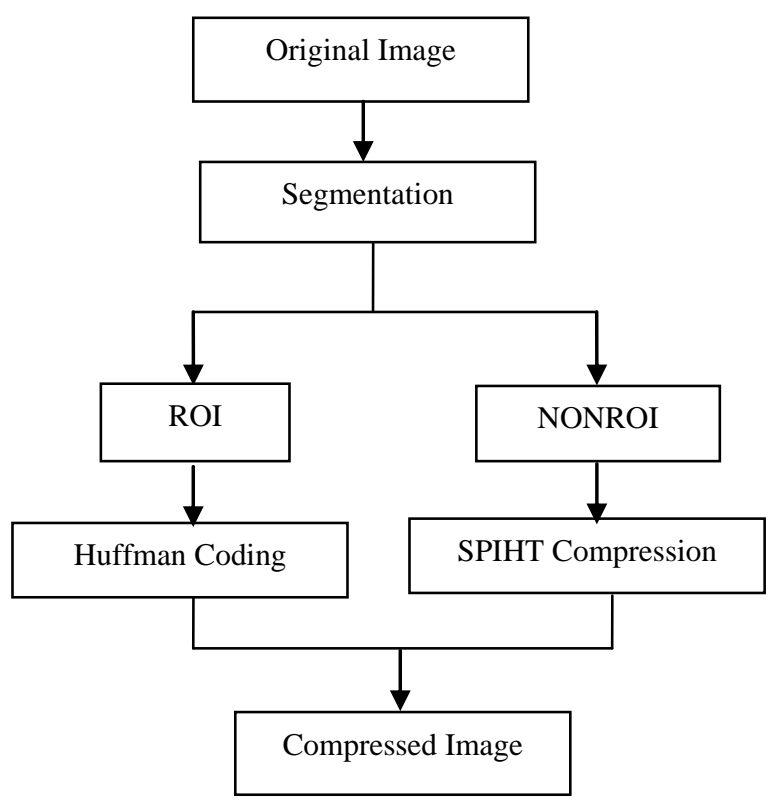

Fig 2: Compression Process

\subsection{Compression of ROI}

The separated tumor region is compressed by lossless Huffman coding technique. Encoding techniques such as LZW, arithmetic coding gives better performance as compared to Huffman coding. However, as the size of cropped tumor region is very small compared to the original image, Huffman coding performs equally efficiently as compared to other encoding techniques [19].

Huffman is a variable length coding algorithm. It requires image sequence, symbols and probability of occurrence of each symbol [20]. Each symbol of uncompressed data is replaced by a code. The term variable length coding indicates that the codes are assigned based on the frequency of occurrence of each symbol. Symbols with higher probability are coded with less number of bits than those that occur less frequently. The variable length codes assigned to input symbols are prefix codes [21]. Huffman coding is a form of entropy coding meaning that the theoretical upper limit of the compression is first order entropy [22].

\subsection{Compression of NONROI}

The image other than region of interest is called background or NONROI. This background is compressed using a lossy SPIHT algorithm. First the image is decomposed into different subbands using biorthogonal type 4.4 wavelet. This wavelet produces highest PSNR values and close correlation among all the wavelets available. The results produced by this 
wavelet are also subjectively efficient [23]. The decomposition level of subbands is kept as a function of image size. SPIHT algorithm is then applied to these decomposed subbands [24].

The compressed bit streams of ROI and NONROI images can be then combined for transmission or storage purpose. The encoded data contains ROI bit stream followed by NONROI bit stream. At the decompression site, the received bit stream is first decomposed into ROI and NONROI compressed files. A respective decompression algorithm is applied to both the files. The images after decompression are merged together to obtained the single compressed image. The flow of the decompression algorithm is depicted in figure 3.The quality of the reconstructed images is verified by various quality metrics.

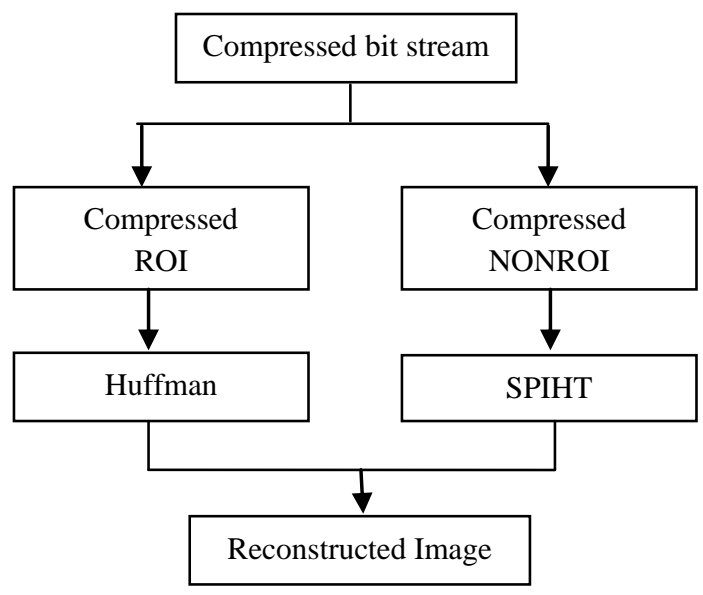

Fig 3: Decompression Process

\subsection{Performance Metrics}

\section{PSNR}

Peak Signal to Noise Ratio (PSNR) is the ratio between the maximum possible power of a signal and the power of corrupting noise that affects the fidelity of its representation. It is measured in terms of Mean Square Error (MSE) as:

$$
\begin{gathered}
P S N R=10 \log \left(\frac{255^{2}}{M S E}\right) \\
M S E=\frac{1}{M \times N} \sum_{m=0}^{M} \sum_{n=0}^{N}(f(m, n)-g(m, n))^{2}
\end{gathered}
$$

where $\mathrm{M} \times \mathrm{N}$ is a size of an image.

\section{SSIM}

The Structural Similarity Index (SSIM) index predicts the perceived quality of digital images. It measures the similarity between two images. The SSIM is modeled considering three different factors such as local luminance similarity, local contrast sensitivity, and local structure similarity which are based on weighted local image statistics [25].

$$
\operatorname{SSIM}=\frac{\left(2 \mu_{x} \mu_{y}+C_{1}\right)\left(2 \sigma_{x y}+C_{2}\right)}{\left(\mu_{x}{ }^{2}+\mu_{y}{ }^{2}+C_{1}\right)\left(\sigma_{x}{ }^{2}+\sigma_{y}{ }^{2}+C_{2}\right)}
$$

Where, $\mu \mathrm{x}, \mu \mathrm{y}$ are local means of luminance, and $\sigma \mathrm{x}, \sigma \mathrm{y}$ are standard deviations of luminance for original and reconstructed images. $\mathrm{C} 1$ and $\mathrm{C} 2$ are the contrast values of the original and reconstructed images.

\section{FSIM}

Feature Similarity Index (FSIM) is a full reference human visual system based image quality assessment model which analyze image based on low level features such as phase congruency (PC) and gradient magnitude (GM). Phase congruency is contrast invariant and measures the local structure. It defines features at point where the Fourier components are having phase maxima. A 2D phase congruency can be calculated using gabor or log gabor filters. Log gabor filters are preferred as they are more capable of encoding natural images with no DC component. Gradient magnitude is used as secondary measure to obtain the contrast information. The FSIM index of an image is given as [26]:

$$
F S I M=\frac{\sum_{x \in \Omega} S_{L}(x) \cdot P C_{\max }(x)}{P C_{\max }(x)}
$$

Where $\mathrm{PCmax}(\mathrm{x})=\max \left(\mathrm{PC}_{1}(\mathrm{x}), \mathrm{PC}_{2}(\mathrm{x})\right)$,

PC1 and PC2 are the phase congruency maps for reference image and distorted image. SL(x) is the combined similarity between reference and distorted image. It is defined as a product of similarity terms of PC and GM.

\section{VIF:}

VIF is a full reference quality assessment metrics which quantifies the mutual information between two quantities. First, the mutual information between input and output of the HVS channel when there is no distortion called as reference image information and second is the mutual information between the input of the distortion channel and the output of HVS channel for the test image. The source or reference image is modeled by wavelet domain Gaussian Scale Mixture. The distortion model is described locally as a combination of uniform wavelet domain energy attenuation with subsequent independent additive noise [27]. Based on the statistical models of source, channel distortion and the receiver, VIF is calculated as:

$$
V I F=\frac{\text { Distorted Image Information }}{\text { Reference Image Information }}
$$

VIF can be calculated either in wavelet domain or pixel domain. In the proposed work, pixel domain model is used.

\section{BRISQUE}

Blind/ Referenceless Image Spatial Quality Evaluator (BRISQUE) is a natural scene statistics based, distortion generic no reference index which operates in spatial domain. A pairwise product of locally normalized luminance values are used to determine the losses in an image. It does not require prior knowledge of the distortions present in an image. As there is no transformation in any other domain, BRISQUE has low computational complexity [28].

It involves three stages:

1. Computation of locally normalized luminance coefficients

2. Modeling of statistical relationship between neighboring pixels.

3. Mapping from feature space to quality score.

The luminance coefficients obtained are Mean Subtracted Contrast Normalized (MSCN) coefficients which are obtained using Generalized Gaussian Distribution (GGD). The features 
are extracted by product of neighboring MSCN coefficients in four directions namely horizontal, vertical, main diagonal and secondary diagonal. These products follow an Asymmetric Generalized Gaussian Distribution (AGGD). The features obtained by multiplication of MSCN coefficients are then mapped into quality score using Support Vector Machine (SVM) regression. The range of BRISQUE varies from 0 to 100. 0 is considered to be the best quality and 100 represents worst quality [30].

\section{SIMULATION RESULTS}

The results obtained from experimentation to test the performance of hybrid coding scheme of SPIHT with Huffman coding are presented in this section. The simulation is carried out in MATLAB environment. T1 and T2 weighted MRI images are used for the simulation purpose. Figure 4 shows the images for which results of simulation are presented.

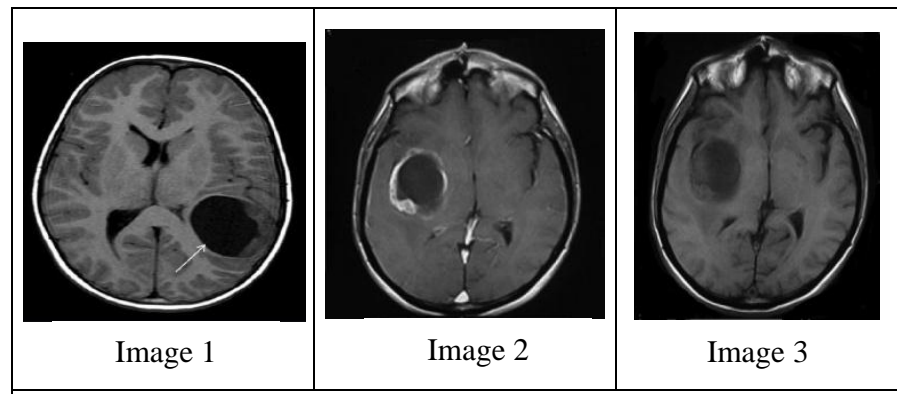

(a) T1 Weighted MRI Images

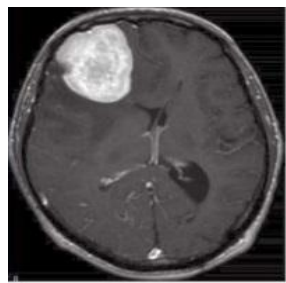

Image 4

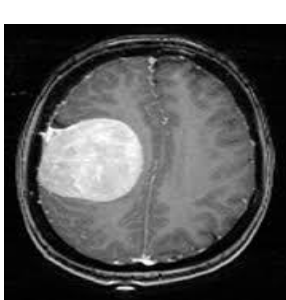

Image 5

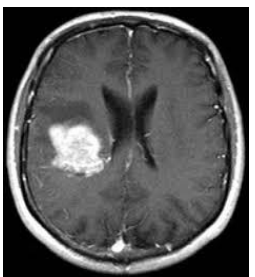

Image 6 (b) T2 Weighted MRI Images

Fig 4 MRI images used for simulation purpose

The results of compression algorithm for T2 weighted MRI image are depicted in figure 5. Figure 5 (a) represents the original image. The binary mask generated using circular window for extraction of ROI is shown in figure 5(b). The tumor region and background is separated by cropping method as shown in figure 5 (c) and (d).

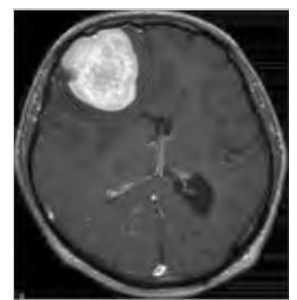

(a)

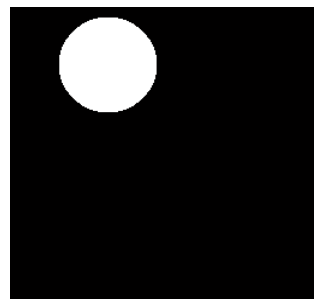

(b)

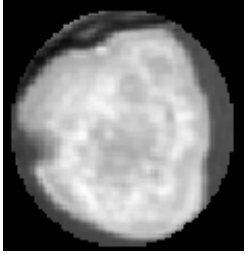

(c)

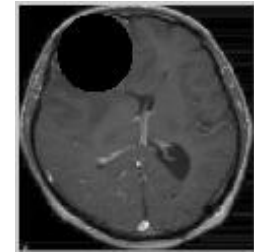

(d)

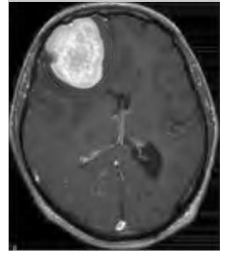

(e)
Fig 5 (a) Original image (b) Circular mask (c) ROI (d) NONROI (e) Reconstructed image

The compression results are shown in table 1 . The amount of compression occurred is calculated in terms of bits per pixel which is the ratio of number of bits after compression to the number of bits before compression.

Table 1 Compression ratios for different images

\begin{tabular}{|c|c|}
\hline Image & Compression ratio (bpp) \\
\hline 1 & 0.0859 \\
\hline 2 & 0.0916 \\
\hline 3 & 0.0861 \\
\hline 4 & 0.1228 \\
\hline 5 & 0.1607 \\
\hline 6 & 0.0926 \\
\hline
\end{tabular}

From table 1, it is observed that the compression depends upon size of cropped ROI. The number of bits per pixel required to represent images increases with increase in size of cropped region. This shows the dependency of compression on extent to which the tumor is spread. As can be seen from figure 4 that image 5 has largest tumor size, it requires more bits per pixel.

The decompressed ROI and NONROI regions are then combined together to obtained final compressed image shown in figure 5 (e). The quality of reconstructed image is evaluated in terms of full reference parameters in table 2 and 3. Table 4 indicates the performance of hybrid scheme for no reference parameter.

Table 2 PSNR performance of proposed hybrid technique

\begin{tabular}{|c|c|c|}
\hline \multirow{2}{*}{ Image } & \multicolumn{2}{|c|}{ PSNR (dB) } \\
\cline { 2 - 3 } & SPIHT & $\begin{array}{c}\text { Proposed } \\
\text { Method }\end{array}$ \\
\hline 1 & 30.40 & 35.01 \\
\hline 2 & 34.48 & 37.68 \\
\hline 3 & 34.20 & 37.27 \\
\hline 4 & 34.23 & 37.74 \\
\hline 5 & 33.06 & 36.44 \\
\hline 6 & 31.93 & 35.40 \\
\hline
\end{tabular}

Table 2 shows higher PSNR values for the proposed hybrid compression scheme over standard SPIHT algorithm. An average of $4.02 \mathrm{~dB}$ improvement is observed for $\mathrm{T} 1$ weighted MRI images while it is $3.40 \mathrm{~dB}$ for T2 weighted MRI images.

Table 3 Performance of proposed hybrid compression scheme in terms of full reference parameters

\begin{tabular}{|c|c|c|c|c|c|c|}
\hline \multirow{2}{*}{$\begin{array}{c}\text { Ima } \\
\text { ge }\end{array}$} & \multicolumn{2}{|c|}{ SSIM } & \multicolumn{2}{c|}{ VIF } & \multicolumn{2}{c|}{ FSIM } \\
\cline { 2 - 7 } & $\begin{array}{c}\text { SPIH } \\
\mathrm{T}\end{array}$ & $\begin{array}{c}\text { Propos } \\
\text { ed } \\
\text { Metho } \\
\text { d }\end{array}$ & $\begin{array}{c}\text { SPIH } \\
\mathrm{T}\end{array}$ & $\begin{array}{c}\text { Propos } \\
\text { ed } \\
\text { Metho } \\
\mathrm{d}\end{array}$ & $\begin{array}{c}\text { SPIH } \\
\mathrm{T}\end{array}$ & $\begin{array}{c}\text { Propos } \\
\text { ed } \\
\text { Metho } \\
\mathrm{d}\end{array}$ \\
\hline 1 & $\begin{array}{c}0.830 \\
7\end{array}$ & 0.9239 & $\begin{array}{c}0.393 \\
8\end{array}$ & 0.4133 & $\begin{array}{c}0.905 \\
9\end{array}$ & 0.9088 \\
\hline
\end{tabular}




\begin{tabular}{|c|c|c|c|c|c|c|}
\hline 2 & $\begin{array}{c}0.925 \\
2\end{array}$ & 0.9596 & $\begin{array}{c}0.480 \\
5\end{array}$ & 0.5019 & $\begin{array}{c}0.933 \\
1\end{array}$ & 0.9360 \\
\hline 3 & $\begin{array}{c}0.904 \\
9\end{array}$ & 0.9523 & $\begin{array}{c}0.441 \\
6\end{array}$ & 0.4590 & $\begin{array}{c}0.983 \\
5\end{array}$ & 0.9309 \\
\hline 4 & $\begin{array}{c}0.899 \\
8\end{array}$ & 0.9528 & $\begin{array}{c}0.401 \\
0\end{array}$ & 0.4212 & $\begin{array}{c}0.938 \\
9\end{array}$ & 0.9444 \\
\hline 5 & 08908 & 0.9460 & $\begin{array}{c}0.506 \\
5\end{array}$ & 0.5182 & $\begin{array}{c}0.933 \\
5\end{array}$ & 0.9368 \\
\hline 6 & $\begin{array}{c}0.874 \\
5\end{array}$ & 0.9357 & $\begin{array}{c}0.386 \\
1\end{array}$ & 0.4115 & $\begin{array}{c}0.933 \\
1\end{array}$ & 0.9358 \\
\hline
\end{tabular}

[5] Vijayvargiva, Gaurav, Sanjay Silakari, and Rajeev Pandev. "A Survev: Various techniques of image compression." arXiv preprint arXiv:1311.6877(2013).

[6] Vemuri, B. C., S. Sahni, F. Chen, C. Kapoor, C. Leonard, and J. Fitzsimmons. "Lossless image compression." (2007).

[7] Erickson, Bradley J. "Irreversible compression of medical images." Journal of Digital Imaging 15, no. 1 (2002): 5-14

SSIM, VIF and FSIM relate the quality analysis of image to HVS system. For an image to have a good perceptual quality, these values should be approaching unity. From table 3, it is observed that the values of SSIM are in the range of 0.92 to 0.96 and that of FSIM are varied from 0.90 to 0.95 for the proposed hybrid technique. The values are near to optimum value. VIF values are not very close to unity, but they are higher than standard SPIHT algorithm which indicates the improved subjective quality of proposed hybrid technique.

Table 4 Performance of proposed hybrid compression scheme in terms of no reference parameters

\begin{tabular}{|c|c|c|}
\hline \multicolumn{3}{|c|}{ BRISQUE } \\
\hline Image & SPIHT & $\begin{array}{c}\text { Proposed } \\
\text { Method }\end{array}$ \\
\hline 1 & 41.970 & 38.4861 \\
\hline 2 & 34.3145 & 31.0343 \\
\hline 3 & 42.2201 & 41.0894 \\
\hline 4 & 67.7482 & 63.4089 \\
\hline 5 & 42.7898 & 41.6089 \\
\hline 6 & 45.7538 & 41.5313 \\
\hline
\end{tabular}

The quality assessment in terms of no reference BRISQUE parameter is presented in table 4. A reduction in the range of 1.1307 to 4.4393 is observed. Decreased values of BRISQUE parameter indicate quality improvement for the proposed for the hybrid technique hybrid technique.

\section{CONCLUSION}

Region based hybrid compression techniques efficiently compresses medical images while retaining the quality of diagnostically important region. A circular window is used to select the tumor region. As the tumor can have arbitrary shape, a circular window proves to be a better choice for extraction of ROI than rectangular window or polygonal window. It provides selection of ROI with less background present. Huffman coding performs as efficiently as other encoding techniques as the size of extracted ROI is less than half of the original image. The compression achieved by hybrid technique is around 64\% less compared to that provided by SPIHT alone. However, higher values of distortion metrics shows improved objective as well as subjective quality of the compressed image. The technique can be incorporated with other compression algorithms for further enhancement in compression.

\section{REFERENCES}

[1] Wei, Wei-Yi. "An introduction to image compression." National Taiwan University, Taipei, Taiwan, ROC (2008).

[2] R.C. Gonzalez and R.E. Woods, Digital Image Processing, Pearson Prentice Hall, third edition, 2008.

[3] https://en.wikipedia.org/wiki/Redundancy_(information theory)

[4] Sonal, Dinesh Kumar. "A study of various image compression techniques."COIT, RIMT-IET. Hisar (2007).

[8] Dhawan, Sachin. "A review of image compression and comparison of its algorithms." International Journal of electronics \& Communication technology2, no. 1 (2011): 22-26.

[9] Erickson, Bradley J., Armando Manduca, Patrice Palisson, Kenneth R. Persons, F. Earnest 4th, Vladimir Savcenko, and Nicholas J. Hangiandreou. "Wavelet compression of medical images." Radiology 206, no. 3 (1998): 599-607.

[10] S Jayaraman, S Esakkirajan, T Veerakumar, Digital Image Processing, Tata McGraw Hill Education pvt. Ltd., Second reprint, 2010.

[11] Walker, James S., and Truong O. Nguven. "Waveletbased image compression." Sub-chapter of CRC Press book: Transforms and Data Compression (2001).

[12] https://en.wikipedia.org/wiki/Medical_imaging

[13] Iniewski, Krzysztof, ed. Medical imaging: Principles, detectors, and Electronics. John Wiley \& Sons, 2009.

[14] Tonarelli, Lorena. "Magnetic resonance imaging of brain tumor."CEwebsource. com (2013).

[15] Jangbari, Palak, and Dhruti Patel. "Review on Region of Interest Coding Techniques for Medical Image Compression." International Journal of Computer Applications 134, no. 10 (2016): 1-5.

[16] Brindha, B., and G. Raghuraman. "Region based lossless compression for digital images in telemedicine application." In Communications and Signal Processing (ICCSP), 2013 International Conference on, pp. 537540. IEEE, 2013.

[17] Rehna, V. J., and M. K. Jeya Kumar. "Hybrid approach to image coding: A review." International Journal of Advanced Computer Science and Applications 2, no. 7 (2011).

[18] Saluia, Nitin, Anoop Kumar, Dr Amisha, and Raiesh Khanna. "CROPPING IMAGE IN RECTANGULAR, CIRCULAR, SQUARE AND TRIANGULAR FORM USING MATLAB."

[19] Somefun Olawale M., Adebayo Adewale O. "Evaluation of Dominant Text Data Compression Techniaues." International Journal of Application or Innovation in Engineering and Management 3,no.6 (2014).

[20] Sharma, Mamta. "Compression using Huffman coding." IJCSNS International Journal of Computer Science and Network Security 10, no. 5 (2010): 133-141.

[21] Mathur, Mridul Kumar, Seema Loonker, and Dheerai Saxena. "Lossless Huffman coding technique for image compression and reconstruction using binary trees." International Journal of Computer Technology and Applications3, no. 1 (2012).

[22] Tavakoli, Nassrin. "Lossless compression of medical images." In Computer-Based Medical Svstems, 1991. Proceedings of the Fourth Annual IEEE Symposium, pp. 200-207. IEEE, 1991.

[23] Sapkal, A. M., and V. K. Bairagi. "Selection of wavelets for medical image compression." International Level IEEE Explorer, IEEE Sponsored ACT2009 (28-29 Dec. 2009) (2009).

[24] Said, Amir, and William A. Pearlman. "A new, fast, and efficient image codec based on set partitioning in hierarchical trees." IEEE Transactions on circuits and systems for video technology 6, no. 3 (1996): 243-250. 
[25] Wang. Zhou, Alan C. Bovik, Hamid R. Sheikh, and Eero P. Simoncelli. "Image quality assessment: from error visibility to structural similarity." IEEE transactions on image processing 13, no. 4 (2004): 600-612.

[26] Zhang, Lin, Lei Zhang, Xuanqin Mou, and David Zhang. "FSIM: a feature similarity index for image quality assessment." IEEE transactions on Image Processing 20, no. 8 (2011): 2378-2386.
[27] Wang. Zhou, and Alan C. Bovik. "A universal image quality index." IEEE signal processing letters 9 , no. 3 (2002): 81-84.

[28] Mittal, Anish, Anush K. Moorthy, and Alan C. Bovik. "Blind/referenceless image spatial quality evaluator." In 2011 Conference Record of the Fortv Fifth Asilomar Conference on Signals, Systems and Computers (ASILOMAR), pp. 723-727. IEEE,2011. 